\title{
Improving the Thermostability and Catalytic Activity of an Inulosucrase by Rational Engineering for the Biosynthesis of Microbial Inulin
}

Dawei $\mathrm{Ni}^{\dagger}$, Shuqi Zhang ${ }^{\dagger}$, Onur Kırtel ${ }^{\ddagger}$, Wei Xu ${ }^{\dagger}$, Qiuming $\mathrm{Chen}^{\dagger}$, Ebru Toksoy Öner ${ }^{\ddagger}$, Wanmeng $\mathrm{Mu}^{*},+, \S$

${ }^{\dagger}$ State Key Laboratory of Food Science and Technology, Jiangnan University, Wuxi, Jiangsu 214122, China

* IBSB-Industrial Biotechnology and Systems Biology Research Group, Department of Bioengineering, Marmara University, Göztepe Campus, 34722 Istanbul, Turkey

§ International Joint Laboratory on Food Safety, Jiangnan University, Wuxi, Jiangsu 214122, China

${ }^{*}$ Corresponding author. Tel.: +86 51085919161 . Fax: +8651085919161

E-mail address: wmmu@jiangnan.edu.cn (W. Mu) 
Table S1. Primers for the construction of Single-point variants using Laga-IS $\Delta 138-702$ as the template

\begin{tabular}{|c|c|c|}
\hline Variants & Primers & Sequences $\left(5^{\prime} \rightarrow 3^{\prime}\right)$ \\
\hline \multirow{2}{*}{ E263D } & E263D-F & GAAGACTTAGACATTTGGGATTCATGGCC \\
\hline & E263D-R & ATCCCAAAT $\underline{G T C T A A G T C T T C T A C T T T T C C T G ~}$ \\
\hline \multirow{2}{*}{ Q332E } & Q332E-F & CCAGTTATTCAAGAATGGTCTGGTT \\
\hline & Q332E-R & CATTGTTGAATAACTGGAGTACCTAAGC \\
\hline \multirow{2}{*}{ Y348F } & Y348F-F & CAATCCAACTTTTCTACACTAAGGTTGATACAAGT \\
\hline & Y348F-R & AGTGTAGAAAAGTTGGATTGAACCATCTTTATTT \\
\hline \multirow{2}{*}{ D424E } & D424E-F & GCACACGTAATTGAAGATAAGGATGGTAATCGT \\
\hline & D424E-R & TCCTTATCTTCAATTACGTGTGCATCACGC \\
\hline \multirow{2}{*}{ A446E } & A446E-F & ATTATCAAGGTGAGGACCAAATTTATC \\
\hline & A446E-R & 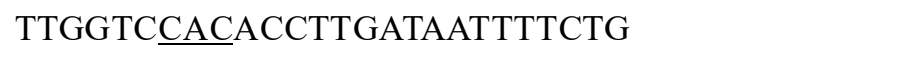 \\
\hline \multirow{2}{*}{ Q451N } & Q451N-F & GACCAAATTTATAACTGGTTAAATTATGGTGGTACT \\
\hline & Q451N-R & TTTAACCAGTTATAAATTTGGTCAGCACCTTGAT \\
\hline \multirow{2}{*}{ S482A } & S482A-F & GCAAAGTGG $\underline{ }$ \\
\hline & S482A-R & CAGCGTTAGCCCACTTTGCTCTATCTTTAATATC \\
\hline \multirow{2}{*}{ R524K } & R524K-F & CTGATGTAGTTAAGTTAGGCAATAAATATTACTT \\
\hline & R524K-R & TATTGCCTAACTTAACTACATCAGGACGTT \\
\hline \multirow{2}{*}{ V572K } & V572K-F & CTCATGGATATAAGCCATTAAACGAATC \\
\hline & V572K-R & TTAATGGCTTATATCCATGAGTTAAGTTATCAG \\
\hline \multirow{2}{*}{ I614M } & I614M-F & CTAT $\underline{A T G A C T A A C C G T G G T G A A G T T ~}$ \\
\hline & I614M-R & CGGTTAGTCATATAGGAAGTAATCAAT \\
\hline \multirow{2}{*}{ A627S } & A627S-F & GTATGCACTCAACTTGGGCACCAAGTTTCT \\
\hline & A627S-R & CAAGTTGAGTGCATACCCTTTCCAGCAACTTC \\
\hline
\end{tabular}


Table S2. Primers for the construction of combinational variants

\begin{tabular}{|c|c|c|c|}
\hline Templates & Variants & Primers & Sequences $\left(5^{\prime} \rightarrow 3^{\prime}\right)$ \\
\hline \multirow{6}{*}{ A446E } & \multirow{2}{*}{ M21 } & S482A-F & GCAAAGTGGGCTAACGCTGCAATCG \\
\hline & & S482A-R & CAGCGTTAGCCCACTTTGCTCTATCTTTAATATC \\
\hline & \multirow{2}{*}{ M22 } & I614M-F & CTATATGACTAACCGTGGTGAAGTT \\
\hline & & I614M-R & CGGTTAGTCATATAGGAAGTAATCAAT \\
\hline & \multirow{2}{*}{ M23 } & A627S-F & GTATGCACTCAACTTGGGCACCAAGTTTCT \\
\hline & & A627S-R & CAAGTTGAGTGCATACCCTTTCCAGCAACTTC \\
\hline \multirow{4}{*}{ S482A } & \multirow{2}{*}{ M24 } & I614M-F & CTATATGACTAACCGTGGTGAAGTT \\
\hline & & I614M-R & CGGTTAGTCATATAGGAAGTAATCAAT \\
\hline & \multirow{2}{*}{ M25 } & A627S-F & GTATGCACTCAACTTGGGCACCAAGTTTCT \\
\hline & & A627S-R & CAAGTTGAGTGCATACCCTTTCCAGCAACTTC \\
\hline \multirow{2}{*}{$\mathrm{I} 614 \mathrm{M}$} & \multirow{2}{*}{ M26 } & A627S-F & GTATGCACTCAACTTGGGCACCAAGTTTCT \\
\hline & & A627S-R & CAAGTTGAGTGCATACCCTTTCCAGCAACTTC \\
\hline \multirow{2}{*}{ M21 } & \multirow{2}{*}{ M31 } & I614M-F & CTATATGACTAACCGTGGTGAAGTT \\
\hline & & I614M-R & CGGTTAGTCATATAGGAAGTAATCAAT \\
\hline \multirow{2}{*}{ M21 } & \multirow{2}{*}{ M32 } & A627S-F & GTATGCACTCAACTTGGGCACCAAGTTTCT \\
\hline & & A627S-R & CAAGTTGAGTGCATACCCTTTCCAGCAACTTC \\
\hline \multirow{2}{*}{ M24s } & \multirow{2}{*}{ M33 } & A627S-F & GTATGCACTCAACTTGGGCACCAAGTTTCT \\
\hline & & A627S-R & CAAGTTGAGTGCATACCCTTTCCAGCAACTTC \\
\hline \multirow{2}{*}{ M31 } & \multirow{2}{*}{ M4 } & A627S-F & GTATGCACTCAACTTGGGCACCAAGTTTCT \\
\hline & & A627S-R & CAAGTTGAGTGCATACCCTTTCCAGCAACTTC \\
\hline \multirow{2}{*}{ M4 } & \multirow{2}{*}{ M5 } & D424E-F & GCACACGTAATTGAAGATAAGGATGGTAATCGT \\
\hline & & D424E-R & TCCTTATCTTCAATTACGTGTGCATCACGC \\
\hline \multirow{2}{*}{ M4 } & \multirow{2}{*}{$\mathrm{M} 4^{\mathrm{N}-33}$} & $\mathrm{~N}-33-\mathrm{F}$ & TATACATATGGCTGAATTAAAGGGCCAAATT \\
\hline & & $\mathrm{N}-33-\mathrm{R}$ & TTAATTCAGCCATATGTATATCTCCTTCTTAAAGTT \\
\hline \multirow{2}{*}{ M5 } & \multirow{2}{*}{ M5 $5^{\mathrm{N}-33}$} & $\mathrm{~N}-33-\mathrm{F}$ & TATACATATGGCTGAATTAAAGGGCCAAATT \\
\hline & & $\mathrm{N}-33-\mathrm{R}$ & TTAATTCAGCCATATGTATATCTCCTTCTTAAAGTT \\
\hline
\end{tabular}




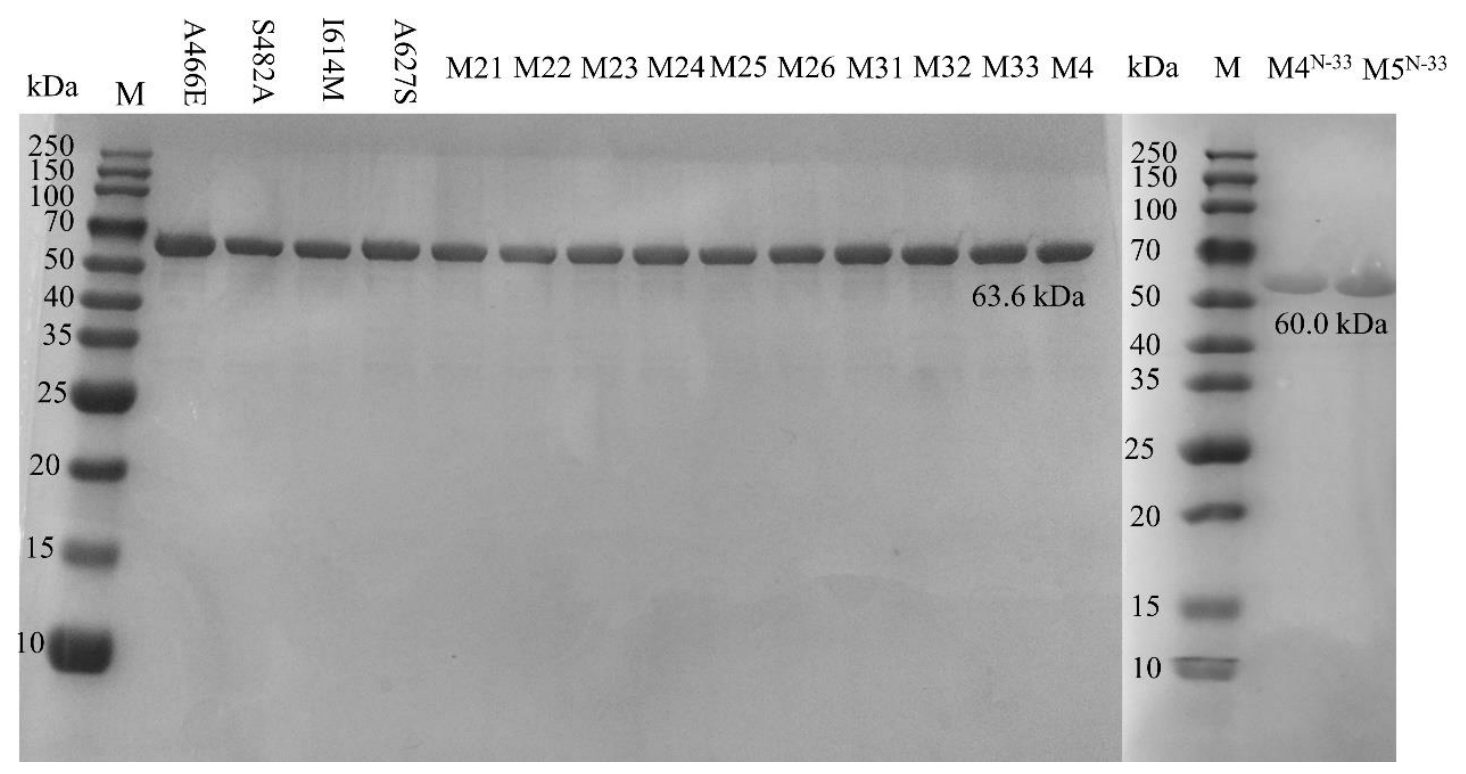

Figure S1. The SDS-PAGE of Laga-IS $\Delta 138-702$ and its various variants. 

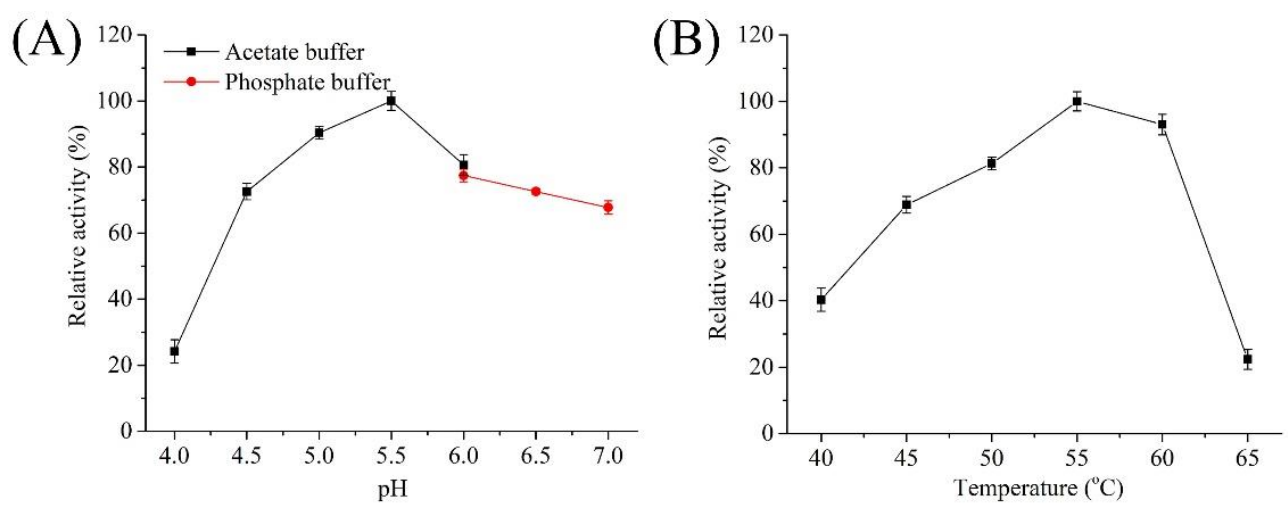

Figure S2. Remeasurement of the optimal $\mathrm{pH}$ and temperature of Laga-IS $\Delta 138-702$.

(A) The effect of $\mathrm{pH}$ on the total activity of Laga-IS $\Delta 138-702$. (B) The effect of on the total activity of Laga-IS $\Delta 138-702$. 


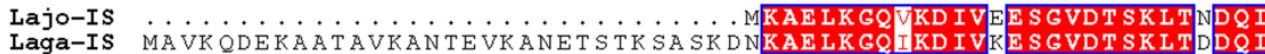

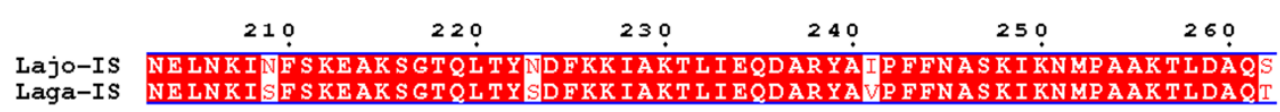

$\begin{array}{llllll}270 & 280 & 290 & 300 & 310 & 320\end{array}$

$\begin{array}{lll}\text { Lajo-IS } & \text { GKVEDLEIWDSWPVQDAKTGYVSNWNGYQLVI GMMGVPNVNDNHIYLLYNKY GDNDFNHW } \\ \text { Laga-IS } & \text { GKVEDLEIWDS WPVQDAKTGYVSNWNGYQLVI GMMGVPNTNDNHIYLLYNKY GDNNFNNW }\end{array}$

$\begin{array}{cccccc} & 330 & 340 & 350 & 360 & 370\end{array}$

Laga-IS KNAGPIFGLGTPVIQOWSGSATLNKDGSIQLYYTKVDTSDNNTNHQKIASATVYLNLEKN

$390 \quad 400 \quad 410 \quad 420 \quad 430 \quad 440$

Lajo-IS QDKI IAHVDNDH IVFEGDGYHYQTYD

Laga-IS QDKISIAHVDNDH IVFEGDGYHYQTYNQWKK TNK GADN IAMRDAHVIDDKDGNRYLVFEA

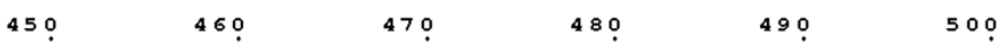

Lajo-IS STGTENYQGDDQ I YQWLNYGGTNKDN L GDFFQ I L SN SD I KDRAKW SNAA I G I IKLNDDVK
Laga-IS STGTENYQGADQI YQWLNYGGTNKDNL GDFLQI L SN SD I KDRAKW SNAA I G I K LNNDTK

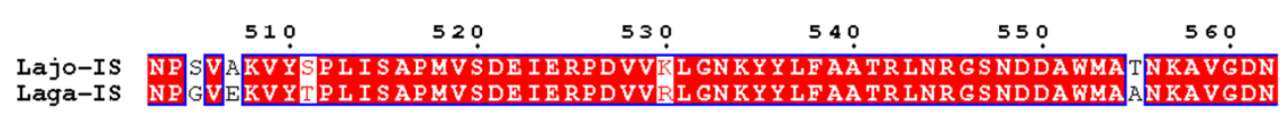

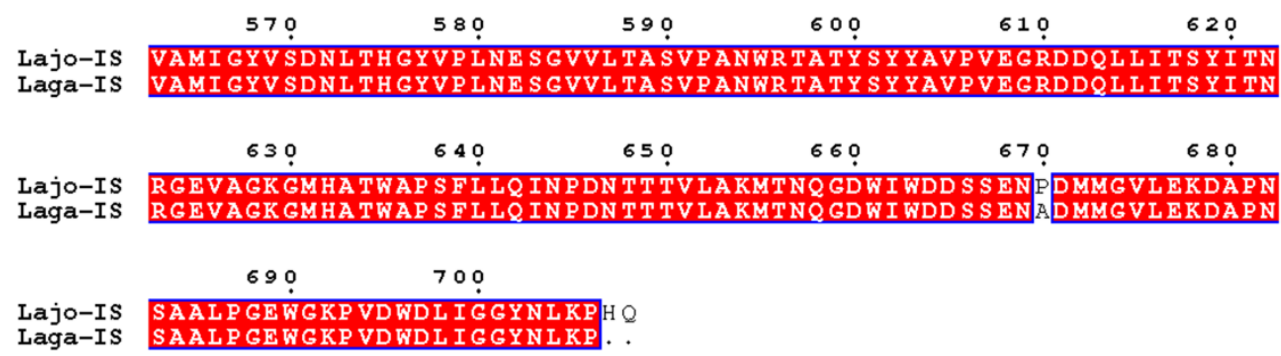

Figure S3. The multiple sequence alignment of Lajo-IS $\Delta 176-708$ and Laga-IS $\Delta 138$ - 